\title{
Effect of Laser Irradiance and Fluoride Varnish on Demineralization Around Dental Composite Restorations
}

\author{
Sara Valizadeh', Maryam Rahimi Khub ${ }^{2}$, Nasim Chiniforush ${ }^{3}$, Mohammad-Javad Kharazifard ${ }^{4}$, Sedighe Sadat \\ Hashemikamangar $^{{ }^{*}}$
}

${ }^{1}$ Assistant Professor, Laser Research Center of Dentistry, Dentistry Research Institute, Restorative Dentistry Department, School of Dentistry, Tehran University of Medical Sciences, Tehran, Iran

${ }^{2}$ Dentist, Teharn University of medical Sciences, Tehran, Iran

${ }^{3}$ Assistant Professor, Dental Implant Research Center, Dentistry Research Institute, Tehran University of Medical Sciences, Tehran, Iran

${ }^{4}$ Research Member, Dental Research Center, Dentistry Research Institute, Tehran University of Medical Sciences, Tehran, Iran ${ }^{5}$ Associate Professor, Restorative department, Dental School, Tehran University of Medical Sciences, Tehran, Iran

\author{
*Correspondence to \\ Sedighe Sadat \\ Hashemikamangar, \\ Tel: +989122403863 \\ Email: smhk58950@gmail.com
}

Published online October 3 2020

\begin{abstract}
Introduction: This study aimed to assess the effects of $\mathrm{CO} 2$ and erbium-doped yttrium aluminum garnet (Er:YAG) lasers with and without fluoride varnish on demineralization around composite restorations.

Methods: This in vitro experimental study evaluated 96 extracted human premolars. After preparation and restoration of class $V$ cavities in the buccal surface of the teeth with composite resin, they were randomly divided into 8 groups of control, CO2 laser (L1), CO2 laser-NaF (L1F), NaF-CO2 laser (FL1), Er:YAG laser (L2), Er:YAG laser-NaF (L2F), NaF-Er:YAG laser (FL2) and NaF (F). The entire surface of the teeth, except for the restored cavity in the buccal surface and $1 \mathrm{~mm}$ around the margin, was coated with two layers of nail varnish. The teeth then underwent $\mathrm{pH}$ cycling for 10 days ( 3 hours in demineralizing solution and 21 hours in remineralizing solution) to artificially induce demineralization. The amount of calcium and phosphorous released into the cariogenic solution was quantified using atomic absorption spectroscopy and spectrophotometry. The Vickers hardness tester was used to measure the hardness of the tooth structure adjacent to composite restoration. Data were analyzed using one-way ANOVA and Tukey's test.

Results: The four groups of L1F, FL1, FL2 and L2F showed minimum loss of calcium and phosphorous ions, and the mean hardness of FL1 and FL2 groups was higher than that of other groups.

Conclusion: The CO2 and Er:YAG lasers alone have no significant effect on the resistance of tooth structure to cariogenic solution. However, they can exert a synergistic effect when used along with $\mathrm{NaF}$ varnish. Fluoride varnish applied prior to laser irradiation confers further resistance to the tooth structure and positively affects its hardness.
\end{abstract}

Keywords: Laser; Recurrent Caries; Fluoride; Surface Hardness.

\begin{abstract}
Introduction
Secondary caries is a major cause of the replacement of dental restorations. ${ }^{1}$ The inherent shrinkage of composite restorations creates a gap between the composite resin and tooth structure. ${ }^{2}$ The tooth structure adjacent to the composite restoration is highly susceptible to caries due to incomplete adaptation of composite restoration to cavity walls. ${ }^{3}$ The interface between the tooth structure and composite restoration is not static. ${ }^{4}$ This interface has a complex dynamic status and is in equilibrium with the minerals present in the composition of saliva. Also, it is affected by the rate of porosities in the tooth structure. ${ }^{5}$
\end{abstract}

Areas at the cavosurface margins are demineralized in case of a shift to acidic conditions.

Encouragement and instruction of oral hygiene measures to patients and increasing the fluoride exposure are among the most effective strategies for caries prevention. Aside from the use of fluoridated toothpaste, the application of phosphopeptide-amorphous calcium phosphate and laser irradiation are among the suggested modalities for this purpose. ${ }^{6}$

Varnish application is a caries preventive method that does not require patients' cooperation. One advantage of fluoride varnish is that its substantivity on the enamel

Please cite this article as follows: Valizadeh S, Rahimi Khub M, Chiniforush N, Kharazifard MJ, Hashemikamangar SS. Effect of laser irradiance and fluoride varnish on demineralization around dental composite restorations. J Lasers Med Sci. 2020;11(4):450-455 doi:10.34172/jlms.2020.70. 
surface is higher than that of other types of topical fluoride products such as mouthwashes, gels and toothpaste. ${ }^{7}$ The application of varnishes is faster than gels. Also, they cause less patient discomfort and are more acceptable by patients. $^{8}$ The potential of conventional fluoride products such as sodium fluoride $(\mathrm{NaF})$ in the prevention of dental demineralization is due to the formation of a layer of calcium fluoride that serves as a physical barrier and prevents the contact of acidic agents with the tooth surface. Also, it serves as a reservoir and during acid attacks, it stimulates enamel remineralization by its buffering capacity. Nonetheless, calcium fluoride can be dissolved in acids. Thus, the protective capacity of $\mathrm{NaF}$ is limited and requires repeated applications. ${ }^{9}$

One application of lasers in dentistry is due to its effect on dental hard tissue to increase its resistance to caries. ${ }^{10}$ The type of laser effect on tissues depends on laser parameters and the target tissue. Wavelength, pulse duration, and energy density of each pulse are among the parameters determining the effect of lasers on tissues. ${ }^{11}$

Evidence shows that laser irradiation can prevent mineral loss and demineralization of the tooth structure due to the effect of acids present in the oral environment. ${ }^{12,13}$ Laser irradiation increases the tooth surface temperature. This thermal rise decreases the dissolution of hydroxyapatite crystals. The incomplete degradation of the organic enamel matrix during laser irradiation obstructs the intra- and inter-prismatic spaces. Thus, acids cannot spread into the internal enamel layers and this postpones the demineralization process. ${ }^{14}$

Due to the high absorption of $\mathrm{CO} 2$ and erbium lasers by the enamel and the ability to provide the required temperature to change the enamel structure and lower its solubility, they can be used for caries inhibition. ${ }^{10}$

The erbium-doped yttrium aluminum garnet (Er:YAG) laser is another laser type used in restorative dentistry. The Er:YAG laser is well absorbed by water and hydroxyl ions present in hydroxyapatite. The Er:YAG laser can effectively increase enamel resistance to acids in demineralized lesions. ${ }^{3}$ In the use of this laser for caries prevention, it is highly important to ensure that laser energy is lower than the ablative threshold in order to induce chemical changes in the enamel surface with no morphological damage. ${ }^{15}$

Considering the significance of preservation of dental tissue adjacent to composite restorations and in order to prevent the occurrence of secondary caries, this study aimed to assess the effect of $\mathrm{CO} 2$ and Er:YAG lasers with and without fluoride varnish on the demineralization of tooth structure around composite restorations.

\section{Materials and Methods}

This in vitro experimental study evaluated 96 extracted human premolars with no caries, discoloration, erosion or cracks. The teeth were cleaned and immersed in 0.5\% chloramine $\mathrm{T}$ solution for 1 week for disinfection. The teeth were then stored in sterile distilled water at room temperature until the experiment.

Class V cavities were created on the buccal surface of the teeth measuring $2 \mathrm{~mm}$ in depth, $2 \mathrm{~mm}$ in occlusogingival height and $2 \mathrm{~mm}$ in mesiodistal width using a diamond bur (008) (Teezkavan, Tehran, Iran) with a high-speed handpiece under air and water spray. All cavities were prepared by one person and after 5 preparations the bur was renewed. The gingival margin of the cavity was 1 $\mathrm{mm}$ below the cementoenamel junction while its incisal margin was $1 \mathrm{~mm}$ above it. For the restoration of the cavities, they were first etched with $37 \%$ phosphoric acid (DenFil, Korea) for 15 seconds and were then rinsed for 10 seconds. A cotton pellet was placed in the cavity (to prevent direct exposure of dentin to air and subsequent dehydration of dentinal tubules) and the cavity surface was dried with air spray for 5 seconds. Next, Single Bond (3M ESPE, St. Paul, MN, USA) was applied and thinned with air spray for 5 seconds. It was then light-cured for 20 seconds. Finally, a microhybrid composite (Filtek Z250; $3 \mathrm{M}$ ESPE, St. Paul, MN, USA) was applied to the cavity in two increments and each increment was light-cured for 20 seconds. After the restoration of the cavity, it was polished with polishing discs. For this purpose, polishing points were used for 30 seconds in a circular, interrupted motion in order to prevent overheating (since it would modify the surface of specimens). The teeth were stored in distilled water for about 2 days until their treatment with the laser or fluoride. The same light-curing unit (WoodPecker, China) with a light intensity of $1000 \mathrm{~mW} /$ $\mathrm{cm}^{2}$ was used for all samples, and during curing, the lightcuring unit was adjacent to the cavity surface.

The teeth were then randomly divided into eight groups of control, CO2 laser (L1), CO2 laser-NaF (L1F), NaFCO2 laser (FL1), Er:YAG laser (L2), Er:YAG laser-NaF (L2F), NaF-Er:YAG laser (FL2) and $\mathrm{NaF}(\mathrm{F})$.

No further intervention was performed for the samples in the control group. In the L1 group, the restoration margins were irradiated with the CO2 laser (Deka, Florence, Italy) with a $10.6 \mu \mathrm{m}$ wavelength, $0.4 \mathrm{~W}$ power, $5 \mathrm{~Hz}$ frequency, $10 \mathrm{~ms}$ pulse duration, $1 \mathrm{~mm}$ spot size and $50.95 \mathrm{~W} / \mathrm{cm}^{2}$. The laser was irradiated horizontally from right to left or left to right, and after completion, the next line was started below the last irradiated point in a reverse fashion. The teeth in the L2 group were subjected to Er:YAG laser irradiation (Pluser, Doctor smile, Italy) of their entire surface with $0.3 \mathrm{~W}$ power, $30 \mathrm{~mJ}$ energy, $10 \mathrm{~Hz}$ frequency and $106.15 \mathrm{~W} / \mathrm{cm}^{2}$ with $5 \%$ water and $85 \%$ air (as coolant) for 10 seconds with back and forth motion. The handpiece was moved $4 \mathrm{~mm}$ above the surface. The teeth in group $\mathrm{F}$ were treated with $\mathrm{NaF}$ fluoride varnish. In this group, $5 \% \mathrm{NaF}$ varnish ( $\mathrm{V}$-varnish, Korea) was applied over the tooth surface by a microbrush according to the manufacturer's instructions after drying the surface. Four minutes of time was allowed in order for the varnish to dry and the teeth were then placed in distilled 
water. The teeth in the LF1 group were first subjected to the CO2 laser (as in group L1) and then underwent $\mathrm{NaF}$ application. The teeth in the FL1 group were first treated with $\mathrm{NaF}$ varnish and their surface was then subjected to $\mathrm{CO} 2$ laser irradiation. The samples in the LF2 group, similar to the L2 group, underwent Er:YAG laser irradiation and then $\mathrm{NaF}$ varnish was applied over the surface of the teeth. The teeth in the FL2 group first underwent NAF varnish application and were then subjected to Er:YAG laser irradiation. The entire surface of the samples, except for the restored cavity in the buccal surface and $1 \mathrm{~mm}$ around the margin, was coated with two layers of nail varnish. Next, in order to create artificial carious lesions, the teeth were subjected to $\mathrm{pH}$ cycling for 10 days as follows ${ }^{16}$ :

First, each tooth was separately immersed in $10 \mathrm{~mL}$ of demineralizing solution comprising of $2.9 \mathrm{~g}$ of $\mathrm{NaCl}, 0.12$ $\mathrm{g}$ of $\mathrm{CaCl}_{2}, 0.13 \mathrm{~g}$ of $\mathrm{NaH}_{2} \mathrm{PO}_{4}, 5 \mathrm{cc}$ of NaN4 and $1.5 \mathrm{cc}$ of acetic acid with a $\mathrm{pH}$ of 4 at $37^{\circ} \mathrm{C}$ for 3 hours. ${ }^{17} \mathrm{Next}$, each sample was rinsed with distilled water for 10 seconds. In order to prevent the dilution of the solution, the teeth were dried with absorbent paper. Next, each tooth was immersed in $10 \mathrm{~mL}$ of remineralizing solution composed of $2.91 \mathrm{~g} \mathrm{NaCl}, 0.12 \mathrm{~g} \mathrm{CaCl}_{2}, 0.13 \mathrm{~g} \mathrm{NaH}_{2} \mathrm{PO}_{4}, 5 \mathrm{cc} \mathrm{NaF}$ and $5 \mathrm{cc} \mathrm{NaN} 3$ with a pH of 7 at $37^{\circ} \mathrm{C}$ for 21 hours. ${ }^{18} \mathrm{Next}$, each sample was rinsed with distilled water for 10 seconds. This cycle was repeated for 9 days and on the $10^{\text {th }}$ day, the samples were only immersed in the remineralizing solution for 24 hours. Both solutions contained NaN3, which has an antibacterial effect and prevents bacterial growth in the solutions. During the 10 -day period, the samples were incubated at $37^{\circ} \mathrm{C}$.

After the completion of $\mathrm{pH}$ cycling, the amount of released calcium and phosphorous ions into the demineralizing solution was quantified to determine the resistance of dental hard tissue adjacent to composite restoration to caries. The concentration of calcium was measured according to the standard protocol of atomic absorption spectroscopy (Analytic Jena Contra AA300). All measurements were repeated 3 times and the mean absorption value was calculated and compared with the 5-point calibration curve. In order to eliminate the interference of phosphate, an equal concentration of La (III) was added to all samples and standards. The concentration of phosphorous was measured according to the standard method using spectrophotometry. All measurements were repeated 3 times and the mean absorption was calculated and compared with the 5-point calibration curve.

In order to measure the hardness of the tooth structure adjacent to the composite restoration, the teeth were mounted in auto-polymerizing acrylic resin (Acropars, Tehran, Iran) in such a way that the surface of the samples was above the level of the acrylic. Next, the surface of the samples was uniformly polished with 800, 1000 and 2000grit abrasive papers (Detecco, Iran). The Vickers hardness test (Bareisis, Germany) was performed on all samples. It was performed at the coronal margin with $50 \mu$ distance from the margin using $200 \mathrm{~g}$ force for 10 seconds in 3 areas with $100 \mu \mathrm{m}$ distance from each other. Data were analyzed using one-way ANOVA and Tukey's test

\section{Results}

Table 1 presents the mean and standard deviation of the amount of calcium and phosphorous released into the cariogenic solution and the hardness number of tooth structure adjacent to composite restoration.

The concentration of phosphorous in the cariogenic solution was lower in the L1F, FL1 and L2F groups compared with other groups. Statistical analysis revealed that the L1F group had significant differences with the $\mathrm{C}, \mathrm{L} 2$ and $\mathrm{F}$ groups in this respect $(P<0.05)$. Also, the FL1 group had a significant difference with the L2 group $(P<0.05)$. The FL2 group had no significant difference with other groups in this respect $(P>0.05)$.

The concentration of calcium in the cariogenic solution in the FL2, L1F and L2F groups was lower than that in other groups. Statistical analysis revealed no significant difference between the FL2 group and other groups $(P>0.05)$. The L2F and L1F groups had no significant difference with other groups $(P>0.05)$ except for the control group ( $P=0.028$ and $P=0.009$ respectively).

The mean hardness number of tooth structure was higher in the FL1 and L2F groups compared with other groups. Nonetheless, the FL1 and L2F groups had no significant differences with any other groups except for the L1 group $(P<0.05)$.

\section{Discussion}

This study aimed to assess the effect of $\mathrm{CO} 2$ and Er:YAG lasers with and without fluoride varnish on tooth demineralization in order to decrease the occurrence of secondary caries around composite restorations. In this study, $\mathrm{pH}$ cycling was performed to simulate the oral environment, which included demineralization and remineralization cycles that simulate the changes in the

Table 1. Mean and Standard Deviation of the Amount of Calcium and Phosphorus Released Into the Cariogenic Solution and Hardness Number of Tooth Structure Adjacent to Composite Restoration

\begin{tabular}{lcccc}
\hline & $\mathrm{N}$ & \multicolumn{3}{c}{ Mean $\pm \mathrm{SD}$} \\
\cline { 3 - 5 } & & $\mathrm{Ca}(\mu \mathrm{g} / \mathrm{mL})$ & $\mathbf{P}(\mu \mathrm{g} / \mathrm{mL})$ & Hardness $(\mathrm{MPa})$ \\
\hline $\mathrm{C}$ & 12 & $179.90 \pm 21.26$ & $90.74 \pm 7.87$ & $390.06 \pm 64.47$ \\
$\mathrm{~L}_{1}$ & 12 & $170.75 \pm 19.09$ & $86.94 \pm 7.66$ & $287.57 \pm 31.57$ \\
$\mathrm{~L}_{1} \mathrm{~F}$ & 12 & $146.20 \pm 22.29$ & $78.92 \pm 7.69$ & $382.97 \pm 93.35$ \\
$\mathrm{FL}_{1}$ & 12 & $159.69 \pm 7.01$ & $79.05 \pm 8.27$ & $475.27 \pm 144.43$ \\
$\mathrm{~L}_{2}$ & 12 & $174.90 \pm 27.15$ & $91.16 \pm 7.56$ & $365.95 \pm 113.62$ \\
$\mathrm{~L}_{2} \mathrm{~F}$ & 12 & $144.88 \pm 18.70$ & $82.54 \pm 5.89$ & $351.75 \pm 149.61$ \\
$\mathrm{FL}_{2}$ & 12 & $134.99 \pm 43.55$ & $77.29 \pm 7.82$ & $409.03 \pm 56.08$ \\
$\mathrm{~F}$ & 11 & $162.80 \pm 11.75$ & $88.70 \pm 4.66$ & $334.02 \pm 110.54$ \\
\hline
\end{tabular}


oral environment that occur during the intake of foods and drinks.

Two different methods were used for a more accurate assessment of carious lesions around composite restorations in this study. First, the amount of calcium and phosphorous released into the cariogenic solution was quantified by atomic absorption spectroscopy and spectrophotometry respectively and reported in micrograms/milliliter $(\mu \mathrm{g} / \mathrm{mL})$. The data revealed that the L1F, FL1, FL2 and L2F groups experienced a minimum loss of calcium and phosphorous ions. Next, the hardness of the enamel around the composite restoration was measured using a Vickers hardness tester. The results showed that the mean hardness of the FL1 and FL2 groups was higher than that of other groups. The analysis of the amount of calcium and phosphorous and the microhardness test showed that the groups treated with $\mathrm{NaF}$ lost lower amounts of minerals and their surface hardness was higher than that of the groups not treated with fluoride.

With regard to the effect of fluoride varnish, the results suggest that fluoride varnish can effectively reinforce the enamel against demineralization. Fluoride is the strongest cariostatic agent that can prevent the occurrence of demineralization. Clinical studies have reported that topical fluoride therapy can prevent caries in $20 \%$ to $40 \%$ of the cases. No significant difference has been reported in the efficacy of different forms of fluoride such as varnish, gel or solution. Thus, the selection of the fluoride product of choice depends on the cost, ease of use, patient acceptance and compliance, and safety of the product. ${ }^{19}$

One reason for the application of lasers in dentistry is their effect on dental hard tissue and increasing its resistance to caries. ${ }^{10}$ The mechanisms of the action of lasers on tooth structure can be divided into 3 categories of physical changes, chemical changes, and kinetic changes and polarity of crystals, which occur due to the conversion of laser energy to thermal energy. ${ }^{20}$ Apel et al. ${ }^{6}$ concluded that it is better not to create melting or ablation on enamel when lasers used for caries prevention purposes. Thus, in the current study, sub-ablative laser parameters were used. High-intensity lasers increase the surface temperature of tissue and are used to prevent enamel demineralization. These laser types decrease the solubility of hydroxyapatite and obstruct the interprismatic spaces by melting and recrystallization of enamel prisms on the surface. Moreover, the application of fluoride varnish or fluoride gel prior to irradiation may convert the enamel hydroxyapatite to fluorapatite and decrease enamel solubility as such. It enables the irradiated surface to retain the fluoride ions for a longer period of time compared with non-laser irradiated enamel. ${ }^{21}$

The Er:YAG laser is more commonly used for hard tissue ablation; however, limited studies have demonstrated that this type of laser with low energy can prevent caries. Researchers have reported that the application of the Er:YAG laser with sub-ablative energy along with fluoride therapy can result in the immediate conversion of hydroxyapatite to fluorapatite..$^{22}$

In line with our findings, some studies have shown that the combination of laser irradiation and fluoride therapy can have a synergistic effect on the resistance of tooth structure to acid attacks. ${ }^{15,23}$ It has been reported that an increase in the resistance of tooth structure to acid exposure caused by the laser is greater when it is combined with fluoride therapy. Daily use of fluoride mouthwashes is tiresome for many patients. However, laser therapy is not performed on a daily basis. A combination of fluoride and laser therapy can decrease the application of fluoride and is an easier measure. ${ }^{19}$

Chinelatti et $\mathrm{al}^{24}$ observed that treatment with the Er:YAG laser had no effect on the microhardness of tooth structure. According to Delbem et al, ${ }^{25}$ the application of the Er:YAG laser with low energy only increased the pressure within the enamel due to temperature rise and water evaporation, without the occurrence of melting or recrystallization. Our study showed that the application of the Er:YAG laser with $60 \mathrm{~mJ} / \mathrm{cm}^{2}$ energy did not cause any significant change in tooth resistance to acid attacks compared with the control group, and the hardness of enamel adjacent to the restoration was lower than that in the control group.

The CO2 laser has four major wavelengths of 9.3, 9.6, 10.3 and $10.6 \mu \mathrm{m}$ in an infrared spectrum. The $9.3 \mu \mathrm{m}$ and $9.6 \mu \mathrm{m}$ wavelengths have the highest use for caries prevention due to their better absorption by hydroxyapatite and efficient and fast warming of minerals. ${ }^{26}$

In the present study, the $\mathrm{CO} 2$ laser with the reported parameters did not have significant efficacy in strengthening the enamel adjacent to composite restoration and increasing its resistance to cariogenic solution, which is due to its higher absorption. No sign of overheating or carbonization was noted on the irradiated surfaces and the only visible change was a white appearance due to water loss and dehydration. Further studies are required to assess the thermal effects of the laser parameters used in this study.

Considering the results, it may be concluded that although surface modifications caused by $\mathrm{CO} 2$ laser irradiation decrease the release of calcium and phosphorous ions into the cariogenic solution compared with the control group, they had no acceptable effect on enamel hardness.

In this study, treatment with the $\mathrm{CO} 2$ laser did not significantly prevent demineralization. CO2 laser irradiation with the parameters adopted in this study may cause morphological changes in the surface such as the formation of microcracks and peeling. These defects during laser therapy are due to the fast expansion of water vapor in the organic enamel matrix. The CO2 laser wavelength has a high coefficient of absorption in the enamel so it can melt the enamel surface in low 
powers. ${ }^{21,26}$ Therefore, this type of laser has the potential to destruct the tooth structure. It is assumed that these morphological changes neutralize the enamel hardening effects and thus, no significant change occurs following the application of this type of laser.

As reported in previous studies, laser irradiation can cause a stronger bond between fluoride-containing particles on the enamel surface, leading to lower enamel solubility. ${ }^{19}$ Moreover, our results showed that laser therapy without the application of fluoride can decrease the calcium concentration compared with the control group, although this reduction was not significant.

Mathew et al compared the effects of Er:YAG and CO2 lasers on enamel resistance to acids and concluded that fluoride therapy combined with the application of these lasers has a synergistic effect on the prevention of enamel demineralization. ${ }^{27}$ Liu et $\mathrm{al}^{28}$ evaluated the effect of the $\mathrm{CO} 2$ laser and fluoride on caries prevention and observed that this type of laser had no synergistic effect with fluoride therapy. Delbem et $\mathrm{al}^{25}$ showed that the combined use of Er:YAG laser and fluoride caused the lowest reduction in microhardness in the demineralization cycle.

In the present study, the release of calcium and phosphorous into the cariogenic solution was lower in the L2F and FL2 groups compared with the control and F groups. Also, the mean microhardness of tooth structure in the FL2 and FL1 groups was higher than that in other groups, but these two groups only had a significant difference with the L2 group. The control group had a significant difference only with the L2 group.

Although some studies have shown that morphological changes in the enamel occur following sub-ablative irradiation of the laser, studies regarding the effect of these changes on bacterial adhesion to irradiated or worn enamel surfaces are limited. Moreover, no previous study has assessed the effect of lasers with regard to caries prevention and remineralization of carious lesions. Future studies with longer durations are required to assess the effect of different laser parameters since they can have a significant effect on laser interactions with dental hard tissue. Furthermore, less mineral loss is expected in the clinical setting due to the presence of saliva. Thus, further in vivo studies are needed to assess the efficacy of different fluoride therapy and laser irradiation protocols for the prevention of incipient carious lesions in the oral environment. ${ }^{19}$

\section{Conclusion}

According to the results, CO2 and Er:YAG lasers alone have no significant effect on the resistance of enamel to cariogenic solution. However, when combined with the application of $\mathrm{NaF}$ varnish, they exert a synergistic effect. Also, the efficacy of the Er:YAG laser in strengthening tooth structure was higher than that of the CO2 laser. Fluoride varnish application prior to laser irradiation has greater efficacy in increasing the resistance of tooth structure and positively affects enamel hardness.

\section{Ethical Considerations}

The present study was approved by the ethics committee of Tehran University of Medical Sciences with code IR.TUMS.DENTISTRY.REC.1397.089.

\section{Conflict of Interests}

The authors declare no conflict of interest.

\section{References}

1. Asghar S, Ali A, Rashid S, Hussain T. Replacement of resin-based composite restorations in permanent teeth. $J$ Coll Physicians Surg Pak. 2010;20(10):639-43. doi: 10.2010/ jcpsp.639643.

2. Lima FG, Romano AR, Correa MB, Demarco FF. Influence of microleakage, surface roughness and biofilm control on secondary caries formation around composite resin restorations: an in situ evaluation. J Appl Oral Sci. 2009;17(1):61-5. doi: 10.1590/s1678-77572009000100012.

3. Colucci V, de Souza Gabriel AE, Scatolin RS, Serra MC, Corona SA. Effect of Er:YAG laser on enamel demineralization around restorations. Lasers Med Sci. 2015;30(4):1175-81. doi: 10.1007/s10103-014-1534-3.

4. Hanabusa M, Mine A, Kuboki T, Momoi Y, Van Ende A, Van Meerbeek B, et al. Bonding effectiveness of a new 'multi-mode' adhesive to enamel and dentine. J Dent. 2012;40(6):475-84. doi: 10.1016/j.jdent.2012.02.012.

5. Jokstad A. Secondary caries and microleakage. Dent Mater. 2016;32(1):11-25. doi: 10.1016/j.dental.2015.09.006.

6. Apel C, Meister J, Götz H, Duschner H, Gutknecht N. Structural changes in human dental enamel after subablative erbium laser irradiation and its potential use for caries prevention. Caries Res. 2005;39(1):65-70. doi: $10.1159 / 000081659$.

7. Todd MA, Staley RN, Kanellis MJ, Donly KJ, Wefel JS. Effect of a fluoride varnish on demineralization adjacent to orthodontic brackets. Am J Orthod Dentofacial Orthop. 1999;116(2):159-67. doi: 10.1016/s0889-5406(99)70213-1.

8. Goodis HE, Fried D, Gansky S, Rechmann P, Featherstone JD. Pulpal safety of $9.6 \mu \mathrm{m}$ TEA $\mathrm{CO}_{2}$ laser used for caries prevention. Lasers Surg Med. 2004;35(2):104-10. doi: 10.1002/lsm.20043.

9. Hemmati S, Dehghan H. Effect of diode and $\mathrm{CO}_{2}$ lasers combined with sodium fluoride varnish on microhardness of deciduous enamel. J Mazandaran Univ Med Sci. 2016;26(138):44-54. [In Persian].

10. Davari A, Daneshkazemi A, Assarzadeh H, Shafiee F. Evaluation of Er:YAG laser and fluoride ion effects on the remineralization of enamel white spot lesions. J Mashhad Dent Sch. 2018;42(3):210-20. [In Persian].

11. Niemz MH. Laser-Tissue Interactions. 3rd ed. Berlin: Springer-Verlag; 2007.

12. Chokhachi Zadeh Moghadam N, Seraj B, Chiniforush N, Ghadimi S. Effects of laser and fluoride on the prevention of enamel demineralization: An in vitro study. J Lasers Med Sci. 2018;9(3):177-182. doi: 10.15171/jlms.2018.32.

13. Mirhashemi AH, Hakimi S, Ahmad Akhoundi MS, Chiniforush N. Prevention of enamel adjacent to bracket demineralization following carbon dioxide laser 
radiation and titanium tetra fluoride solution treatment: An in vitro study. J Lasers Med Sci. 2016;7(3):192-196. doi: 10.15171/jlms.2016.33.

14. Nogueira RD, Silva CB, Lepri CP, Palma-Dibb RG, GeraldoMartins VR. Evaluation of surface roughness and bacterial adhesion on tooth enamel irradiated with high intensity lasers. Braz Dent J. 2017;28(1):24-9. doi: 10.1590/01036440201701190.

15. Ghadimi S, Khami MR, Razeghi S. Combined effect of laser irradiation and fluoride application in dental caries prevention. Journal of Dental Medicine. 2015;28(1):76-85. [In Persian].

16. Klein AL, Rodrigues LK, Eduardo CP, Nobre dos Santos M, Cury JA. Caries inhibition around composite restorations by pulsed carbon dioxide laser application. Eur J Oral Sci. 2005;113(3):239-44. doi: 10.1111/j.16000722.2005.00212.x.

17. Ekambaram M, Itthagarun A, King NM. Comparison of the remineralizing potential of child formula dentifrices. Int J Paediatr Dent. 2011;21(2):132-40. doi: 10.1111/j.1365263X.2010.01101.x.

18. Amaechi BT, Higham SM, Edgar WM. Factors affecting the development of carious lesions in bovine teeth in vitro. Arch Oral Biol. 1998;43(8):619-28. doi: 10.1016/s00039969(98)00043-0.

19. Fekrazad R, Ebrahimpour L. Evaluation of acquired acid resistance of enamel surrounding orthodontic brackets irradiated by laser and fluoride application. Lasers Med Sci. 2014;29(6):1793-8. doi: 10.1007/s10103-013-1328-z.

20. Amaechi BT, Higham SM. In vitro remineralisation of eroded enamel lesions by saliva. J Dent. 2001;29(5):371-6. doi: 10.1016/s0300-5712(01)00026-4.

21. Ramalho KM, de Paula Eduardo C, Heussen N, Rocha RG, Lampert F, Apel C, et al. Protective effect of $\mathrm{CO}_{2}$ laser (10.6 $\mu \mathrm{m})$ and fluoride on enamel erosion in vitro. Lasers Med Sci. 2013;28(1):71-8. doi: 10.1007/s10103-012-1071-x.

22. Demito CF, Vivaldi-Rodrigues G, Ramos AL, Bowman SJ. The efficacy of a fluoride varnish in reducing enamel demineralization adjacent to orthodontic brackets: an in vitro study. Orthod Craniofac Res. 2004;7(4):205-10. doi: 10.1111/j.1601-6343.2004.00305.x.

23. Yamamoto H, Sato K. Prevention of dental caries by acoustooptically Q-switched Nd: YAG laser irradiation. J Dent Res. 1980;59(2):137. doi: 10.1177/00220345800590020801.

24. Chinelatti MA, Rocha CT, Colucci V, Serra MC, RodriguesJúnior AL, Corona SA. Effect of Er:Yag laser on dentin demineralization around restorations. Lasers Med Sci. 2017;32(2):413-8. doi: 10.1007/s10103-016-2136-Z.

25. Delbem AC, Cury JA, Nakassima CK, Gouveia VG, Theodoro LH. Effect of Er:YAG laser on CaF2 formation and its anti-cariogenic action on human enamel: an in vitro study. J Clin Laser Med Surg. 2003;21(4):197-201. doi:10.1089/104454703768247765

26. de Melo JB, Hanashiro FS, Steagall W Jr, Turbino ML, Nobre-dos-Santos M, Youssef MN, et al. Effect of $\mathrm{CO}_{2}$ laser on root caries inhibition around composite restorations: an in vitro study. Lasers Med Sci. 2014;29(2):525-35. doi: 10.1007/s10103-012-1259-0.

27. Mathew A, Reddy NV, Sugumaran DK, Peter J, Shameer M, Dauravu LM. Acquired acid resistance of human enamel treated with laser (Er: YAG laser and Co2 laser) and acidulated phosphate fluoride treatment: An in vitro atomic emission spectrometry analysis. Contemp clin dent. 2013 ;4(2):170-5. doi: 10.4103/0976-237X.114864.

28. Liu Y, Hsu CY, Teo CM, Teoh SH. Potential mechanism for the laser-fluoride effect on enamel demineralization. J Dent Res. 2013;92(1):71-5. doi: 10.1177/0022034512466412. 